Proc. 17th Winter Workshop on

Nuclear Dynamics (2001)

17th Winter Workshop on Nuclear Dynamics

Park City, Utah, USA

March 10-17, 2001

\title{
NUC-MINN-01/3-T \\ Dissipative Relativistic Fluid Dynamics for Nuclear Collisions
}

\author{
Azwinndini Muronga \\ School of Physics and Astronomy, University of Minnesota, \\ 116 Church Street S.E., Minneapolis, Minnesota 55455, USA \\ Email address: amuronga@physics.spa.umn.edu
}

\begin{abstract}
In the context of the Müller-Israel-Stewart second-order theory for dissipative fluids due to Grad, we analyze the effects of thermal conduction and viscosity in heavy ion collisions. We contrast the results to those of the first-order theory due to Eckart and to Landau and Lifshitz and to those of perfect (ideal) fluid due to Euler. We study the energy density and entropy density evolution of a pion gas produced in the heavy ion collisions. The truncated version of the second-order theory is used to find the dissipative quantities.

Keywords: relativistic fluid dynamics, relativistic heavy ion collisions, hydrodynamic model, collective flow

PACS: 25.75.Ld, 25.75.-q, 24.10.Jv, 24.10.Nz, 47.75.+f
\end{abstract}

\section{Introduction}

Heavy ion collisions such as those at RHIC provide a basic tool to study the properties of hot and dense matter produced at high energies. Of particular importance is knowledge of the space-time evolution of the matter. This is important when one wants to study the transition from hadron to quark and gluon degrees of freedom, a state of matter known as QGP (quark-gluon plasma), as predicted by QCD [1]. One way to study the dynamics of the produced matter is by using fluid dynamics [ [2, 3]. To probe the non-equilibrium properties of the matter we need to include dissipative effects in the fluid dynamic modeling of heavy ion reactions. It is known even in non-relativistic dissipative fluids that dissipation will have an effect on the observables [ 4 .

The standard theory of dissipative fluid dynamics developed by Eckart [5] and by Landau and Lifshitz [6] exhibits certain undesirable effects. The resulting transport equations of dissipative fluxes lead to parabolic equations for heat conduction and shear diffusion. Thus it predicts an infinite speed of propagation for thermal and viscous signals. Many applications of dissipative fluid dynamics in relativistic nuclear collisions have used the standard theory [7]. Because of such undesirable effects, it is then necessary to apply an- 
other thermodynamic theory of irreversible processes that does not present this anomalous behavior. Causal theory of dissipative fluids developed by Müller [ 14] and by Israel and Stewart [ 15] due to Grad [ 13] were developed to remedy some of these undesirable features. The resulting equations of causal theory are hyperbolic in structure and lead to causal propagation. Thus causal theory seems to be a good candidate to use instead of the standard theory. In the causal-type theories the space of thermodynamic variables is extended to include dissipative flows. These dissipative flows (heat flows and viscous pressures) are considered as independent variables. The entropy four-flow then depends not only on the primary variables (number density, energy density, and pressure) but on these dissipative flows as well and its production is semi-positive definite. At equilibrium, the entropy is maximum.

\section{Basic Features of Relativistic Dissipative Fluid Dynamics}

The basic formulation of relativistic hydrodynamics can be found in literature [ 6, 8, 9 , 10, 11, 12]. We will use the natural units: $\hbar=c=k_{\mathrm{B}}=1$. The metric tensor we use is $g^{\mu \nu}=\operatorname{diag}(+,-,-,-)$. We consider a simple fluid and no electromagnetic fields. This fluid is characterized by,

$$
\begin{aligned}
N_{A}^{\mu}(x) & \text { (particle 4-current), } \\
T^{\mu v}(x) & \text { (energy-momentum tensor) } \\
S^{\mu}(x) & \text { (entropy 4-current) }
\end{aligned}
$$

where $A=1, \ldots, n$ for the $n$ conserved net charge currents, such as electric charge, baryon number, and strangeness. $N^{\mu}$ and $T^{\mu \nu}$ represent conserved quantities:

$$
\begin{aligned}
\partial_{\mu} N_{A}^{\mu} & \equiv 0 \\
\partial_{\mu} T^{\mu v} & \equiv 0
\end{aligned}
$$

The above equations are the local conservation of net charge and energy-momentum. They are the equations of motion of a relativistic fluid. There are $4+n$ equations and $10+4 n$ independent unknown functions. The second law of thermodynamics requires

$$
\partial_{\mu} S^{\mu} \geq 0
$$

and it forms the basis for the development of the extended irreversible thermodynamics.

\subsection{Tensor Decomposition}

We now perform a tensor decomposition of $N_{A}^{\mu}, T^{\mu v}$, and $S^{\mu}$ with respect to an arbitrary, time-like, normalized 4-vector $u^{\mu}, u^{\mu} u_{\mu}=1$. The projection onto the 3 -space orthogonal to $u^{\mu}$ is denoted by

$$
\triangle^{\mu v} \equiv g^{\mu v}-u^{\mu} u^{v}=\triangle^{v \mu}, \triangle^{\mu v} u_{v}=0, \triangle^{\mu \alpha} \triangle_{\alpha}^{v}=\triangle^{\mu v}, \triangle_{v}^{v}=3
$$


The tensor decomposition reads:

$$
\begin{aligned}
N_{A}^{\mu} & =n_{A} u^{\mu}+V_{A}^{\mu}, \\
T^{\mu v} & =\varepsilon u^{\mu} u^{v}-p \triangle^{\mu v}+2 W^{(\mu} u^{v)}+t^{\mu v}, \\
S^{\mu} & =s u^{\mu}+\Phi^{\mu}
\end{aligned}
$$

where we have defined

$$
\begin{aligned}
& W^{\mu}=q^{\mu}+h V^{\mu}, \\
& t^{\mu v}=\pi^{\mu \nu}-\Pi \triangle^{\mu v}
\end{aligned}
$$

and the parenthesis notation is defined by

$$
A^{(\mu v)} \equiv \frac{1}{2}\left(A^{\mu v}+A^{v \mu}\right) .
$$

In this presentation $h$ is the enthalpy per particle defined by

$$
h=\frac{(\varepsilon+p)}{n} .
$$

The dissipative fluxes satisfy the following orthogonality relations:

$$
u_{\mu} V_{A}^{\mu}=0, u_{\mu} q^{\mu}=0, u_{\mu} W^{\mu}=0, u_{\mu} t^{\mu v}=0, \pi_{v}^{v}=0 .
$$

In the local rest frame (LRF) where $u^{\mu}=(1, \overrightarrow{0})$, the quantities appearing in the decomposed tensors have the following meanings:

$$
\begin{aligned}
n_{A} & \equiv u_{\mu} N_{A}^{\mu} & & \text { net density of charge of type } A \\
V_{A}^{\mu} & \equiv \triangle_{v}^{\mu} N_{A}^{v} & & \text { net flow of charge of type } A, \\
\varepsilon & \equiv u_{\mu} T^{\mu v} u_{v} & & \text { energy density }, \\
p+\Pi & \equiv-\frac{1}{3} \triangle_{\mu v} T^{\mu v} & & \text { pressure }, \\
q^{\mu} & \equiv u_{v} T^{v \lambda} \triangle_{\lambda}^{\mu} & & \text { heat flow }, \\
\pi^{\mu v} & \equiv T^{\langle\mu v\rangle} & & \text { stress tensor }, \\
s & \equiv u_{\mu} S^{\mu} & & \text { entropy density } \\
\Phi^{\mu} & \equiv \triangle_{v}^{\mu} S^{v} & & \text { entropy flux } .
\end{aligned}
$$

The angular bracket notation is defined by

$$
A^{<\mu \nu>} \equiv\left[\frac{1}{2}\left(\triangle_{\sigma}^{\mu} \triangle_{\tau}^{v}+\triangle_{\tau}^{\mu} \triangle_{\sigma}^{v}\right)-\frac{1}{3} \triangle^{\mu \nu} \triangle_{\sigma \tau}\right] A^{\sigma \tau} .
$$

The space-time derivative decomposes into

$$
\partial^{\mu}=u^{\mu} D+\nabla^{\mu}, \quad u^{\mu} \nabla_{\mu}=0
$$


where

$$
\begin{aligned}
D & \equiv u^{\mu} \partial_{\mu} & & \text { convective time derivative } \\
\nabla^{\mu} & \equiv \triangle^{\mu v} \partial_{v} & & \text { gradient operator } .
\end{aligned}
$$

In LRF the two operators attain the given meanings. In this rest frame the projector becomes

$$
\triangle_{\mu v}=\triangle^{\mu v}=\operatorname{diag}(0,-1,-1,-1), \quad \triangle_{v}^{\mu}=\operatorname{diag}(0,1,1,1),
$$

and the heat four flow has spatial components only $\left(q^{\mu}=(0, \vec{q})\right)$.

\section{First Set of Equations: The Conservation Laws}

From now on we will consider one type of charge, namely the net baryon number. With the help of the orthogonality properties given in the previous section we obtain the conservation laws. The equation of continuity (baryon conservation), $\partial_{\mu} N^{\mu} \equiv 0$, the equation of motion (momentum conservation), $\triangle_{v}^{\mu} \partial_{\lambda} T^{\nu \lambda} \equiv 0$, and the equation of energy (energy conservation), $u_{\mu} \partial_{v} T^{\mu \nu} \equiv 0$ are respectively given by

$$
\begin{aligned}
D n & =-n \nabla_{\mu} u^{\mu}-\nabla_{\mu} V^{\mu}+V_{\mu} D u^{\mu} \\
(\varepsilon+p+\Pi) D u^{\mu} & =\nabla^{\mu}(p+\Pi)-\nabla_{v} \pi^{\mu v}+\pi^{\mu v} D u_{v}-\left[\triangle_{v}^{\mu} D W^{\mu}+2 W^{(\mu} \nabla_{v} u^{v)}\right] \\
D \varepsilon & =-(\varepsilon+p+\Pi) \nabla_{\mu} u^{\mu}+\pi^{\mu v} \nabla_{v} u_{\mu}-\nabla_{\mu} W^{\mu}+2 W^{\mu} D u_{\mu}
\end{aligned}
$$

There are 5 conservation equations and 14 unknown functions. We need 9 additional equations to close the system. So far $u^{\mu}$ is arbitrary. It can be chosen to be the particle 4-velocity. This is known as the Eckart frame or particle frame. In this frame $V^{\mu} \equiv 0$. Alternatively one can choose it to be the 4-velocity of the energy flow. This is known as the Landau and Lifshitz frame or energy frame. In this frame $W^{\mu} \equiv 0$. Using the fundamental thermodynamic equation of Gibbs the energy balance equation can be written in the following convenient form:

$$
T \partial_{\mu} S^{\mu}=\sigma_{\mu v} \pi^{\mu v}-\Pi \theta-\partial_{\mu} q^{\mu}+q^{\mu} a_{\mu}
$$

where

$$
\begin{aligned}
a_{\alpha} & \equiv u^{\beta} \partial_{\beta} u_{\alpha} & & \text { (4-acceleration of the fluid) } \\
\omega_{\alpha \beta} & \equiv \triangle_{\alpha}^{\mu} \triangle_{\beta}^{v} \frac{1}{2}\left(\partial_{v} u_{\mu}-\partial_{\mu} u_{v}\right) & & \text { (vorticity tensor) } \\
\theta_{\alpha \beta} & \equiv \triangle_{\alpha}^{\mu} \triangle_{\beta}^{v} \frac{1}{2}\left(\partial_{v} u_{\mu}+\partial_{\mu} u_{v}\right) & & \text { (expansion tensor) } \\
\theta & \equiv \triangle^{\alpha \beta} \theta_{\alpha \beta}=\partial_{\alpha} u^{\alpha} & & \text { (volume expansion) } \\
\sigma_{\alpha \beta} & \equiv \theta_{\alpha \beta}-\frac{1}{3} \triangle_{\alpha \beta} \theta & & \text { (shear tensor) }
\end{aligned}
$$




\section{Second Set of Equations: The Transport Equations}

There are two approaches for finding the transport equations in addition to conservation laws. The first one is the phenomenological approach and is based on the second law of thermodynamics, that is, the principle of non-decreasing entropy. The standard theories of dissipative fluid dynamics [5, 6] assume that the entropy four flow is linear in dissipative quantities. Hence they are also referred to as first-order theories. The extended theories allow the inclusion of terms that are quadratic in the dissipative quantities, $\Pi, q^{\mu}$ and $\pi^{\mu \nu}$. They are referred to as second-order theories. Using conservation laws $u_{v} \partial_{\mu} T^{\mu v}=0$ and $\partial_{\mu} N^{\mu}=0$ we then obtain the expression for the entropy production $\partial_{\mu} S^{\mu}$ from which we obtain the transport equations by requiring that the entropy production be positive. The second approach uses kinetic theory and is based on Boltzmann moment equations. The results of transport equations to be presented here are obtained by using a relativistic Grad's 14 moment approximation [ [13]. However, one can also use the first Chapman-Enskog approximation [ 17] to find the transport equations in the Eckart theory. Both the phenomenological and kinetic theory approaches require that the deviations from local thermodynamic equilibrium be small, that is, $V^{\mu}, q^{\mu}, \pi^{\mu \nu}$ and $\Pi$ are small compared to $\varepsilon, p$, and $n$.

In the standard Eckart theory one obtains the following set of transport equations for the bulk viscous pressure, the heat flow and the shear viscous pressure respectively

$$
\begin{aligned}
\Pi & \equiv-\zeta \nabla_{\mu} u^{\mu} \\
q^{\mu} & \equiv \lambda T\left(\frac{\nabla^{\mu} T}{T}-D u^{\mu}\right)=-\lambda n T^{2} \nabla^{\mu}\left(\frac{\mu}{T}\right), \\
\pi^{\mu \nu} & \equiv 2 \eta \nabla^{\langle\mu} u^{v\rangle}
\end{aligned}
$$

where $\zeta(\sigma, \varepsilon, n), \lambda(\sigma, \varepsilon, n)$, and $\eta(\sigma, \varepsilon, n)$ are the bulk viscosity, thermal conductivity and shear viscosity coefficients. These transport coefficients are required to be positive by the second law of thermodynamics

$$
\partial_{\mu} S^{\mu}=\frac{\Pi^{2}}{\zeta T}-\frac{q^{\mu} q_{\mu}}{\lambda T^{2}}+\frac{\pi^{\mu v} \pi_{\mu v}}{2 \eta T} \geq 0 .
$$

The resulting equations of motion are parabolic, unstable under perturbations and lead to an acausal nature of propagation [ 15, 16]. This is a paradox since in special relativity the speed of light is finite and all maximum speeds should not be greater than this speed. This paradox was first addressed by Cattaneo [ 18]. In the above set of equations, if a thermodynamic force is suddenly switched off, then the corresponding flux instantaneously vanishes, indicating that a signal propagates through the fluid at infinite speed, violating relativistic causality. Even in the non-relativistic case, infinite speeds present a problem, since physically we expect the signal speed to be limited by the maximum molecular speed. To avoid this paradox Cattaneo introduced $a d$ hoc relaxation terms in the phenomenological equations. The resulting equations conform with causality and hyperbolicity requirements. The only problem was that a theory developed from first principles was needed. It is from these arguments that the extended theory of Müller, Israel and Stewart was developed. 
The resulting transport equations derived from extended theories differ from those of Eckart-type theories: they contain relaxation terms. These relaxation terms make the structure of the resulting equations hyperbolic and thus will conform with causality requirements. Although it may not be reasonable in some situations, we shall assume here for simplicity that there are no viscous/heat couplings (i.e. $\alpha_{0}=\alpha_{1}=0$ in [ 15] ). We will also assume that there are no couplings of vorticity and acceleration to the heat and shear fluxes. In these approximations we are left with a simple but still causal structure of transport equations which has the Maxwell-Cattaneo [ 18] form of transport equations. The Müller-Israel-Stewart equations reduce, in the Eckart frame, to

$$
\begin{aligned}
\tau_{\Pi} D \Pi+\Pi & \simeq-\zeta \theta, \\
\tau_{q} D q^{\mu}+q^{\mu} & \simeq \lambda T\left(\frac{\nabla^{\mu} T}{T}-D u^{\mu}\right), \\
\tau_{\pi} D \pi^{\mu \nu}+\pi^{\mu \nu} & \simeq 2 \eta \sigma^{\mu \nu},
\end{aligned}
$$

where

$$
\tau_{\Pi}=\zeta \beta_{0}, \quad \tau_{q}=\lambda T \beta_{1}, \quad \tau_{\pi}=2 \eta \beta_{2}
$$

Here the $\beta_{A}(\varepsilon, n)$ are the relaxation coefficients of the dissipative fluxes. As before $\zeta, \lambda, \eta$ are the transport coefficients. They involve complicated collision integrals, and they also depend on the equation of state. The $\tau_{A}$ are the relaxation times. They are sometimes taken to be the collision time $\left(\tau_{c o l} \approx 1 /(n \sigma v)\right.$, with $\sigma$ being the cross section and $v$ the mean particle speed). In general they are different from the collision time. Here $\tau_{A}$ will be taken to be the time for dissipative fluxes to relax to their equilibrium values. The relaxation terms in the extended theories make it possible for one to study the evolution of the dissipative quantities. The transport equations are coupled to the evolution equations for number density, energy density and momentum. The evolution equations together with the transport equations form a quasi-linear, symmetric and hyperbolic system of 14 first order partial differential equations. The system of equations is found to fulfill the requirements of causality and hyperbolicity [ 15, 16]. One also needs to investigate carefully the conditions under which the truncated equations are reasonable.

\section{The equation of state and transport coefficients}

In this presentation we study the energy density and entropy density evolution in the $1+1$ Bjorken hydrodynamic limit [ 19]. We therefore consider equations (31) and (32). The equation of state is that of a massless pion gas. Thus the pressure is given by $p=a T^{4}$ with $a=g_{h} \pi^{2} / 90$ where $g_{h}=3$ is the number of degrees of freedom, the energy density and entropy density are given by $\varepsilon=3 a T^{4}$ and $s=4 a T^{3}$ respectively. From the transport equations, the bulk viscous equation does not contribute for massless particles, $(\zeta \longrightarrow 0)$ [ 8]. For the $1+1$ dimensional Bjorken-type hydrodynamics the heat term in the energy equation will not contribute. Thus we need only the shear viscous pressure for this presentation. The energy density evolution equation (31) becomes

$$
\frac{d \varepsilon}{d \tau}=-\frac{(\varepsilon+p)}{\tau}+\frac{\Phi}{\tau}
$$


where

$$
\begin{aligned}
\Phi & \equiv 0 & & \text { (perfect fluid) } \\
\Phi & =\frac{4}{3} \eta / \tau & & \text { (standard theory) } \\
\tau_{\pi} \frac{d \Phi}{d \tau} & =-\Phi+\frac{4}{3} \eta / \tau & & \text { (extended theory) }
\end{aligned}
$$

where $\eta=b T^{-1}$ [20] and $\left(b=\pi / 8 f_{\pi}^{4}\right)$ where $f_{\pi}=93 \mathrm{MeV}$ is the pion decay constant. For massless particles $\beta_{2}=3 /(4 p)$, and this is used in the expression for $\tau_{\pi}$. The energy equation can be solved analytically for the perfect fluid and first order (provided $\eta$ is constant) cases. But since we want $\eta$ to depend on temperature or time one can then solve the equations numerically or first find the temperature evolution as done in [21]. In the case of the second-order theory we solve the equations numerically. The proper time evolution of energy density is given by

$$
\begin{array}{ll}
\varepsilon(\tau)=\varepsilon\left(\tau_{0}\right)\left[\frac{\tau}{\tau_{0}}\right]^{-4 / 3} \quad \text { (perfect fluid) } \\
\varepsilon(\tau)=\left\{\varepsilon\left(\tau_{0}\right)-4 \eta / \tau_{0}\right\}\left[\frac{\tau}{\tau_{0}}\right]^{-4 / 3}+4 \eta / \tau \quad \text { (first order) }
\end{array}
$$

Here $\tau_{0}$ represents the instant at which the expansion starts. In Figs. 11 through 4 we show the $\tau$ dependence of energy density $\varepsilon$ and entropy density $s$ for the three different cases: a perfect fluid, a first-order theory of dissipative fluids and a second-order theory of dissipative fluids. In Figs. 1 and 2 we show the dependence of the time evolution of energy density on the initial time $\tau_{0}$. In Figs. 3 and 4 we show the dependence of time evolution of entropy density on the initial time $\tau_{0}$. In both cases we take the initial initial temperature to be $200 \mathrm{MeV}$.

First we note that the presence of dissipation makes difference. In the earlier times the first-order (parabolic) theory predicts a peak in the energy density and entropy while the perfect fluid and second-order (both hyperbolic) predict a monotonic decrease. By looking at the dependence of $\varepsilon$ and $s$ on $\tau_{0}$, we see that it is in the early times that the difference is most pronounced. In the later times there is an indication that the two dissipative theories might converge. Therefore knowledge of different time scales is crucial to determine when to apply which theory. In heavy ion reactions, where the dynamics is happening in very short times, we need to use the hyperbolic theories of relativistic dissipative fluid dynamics.

The presence of dissipation in heavy ion reactions will have profound effects on the space-time evolution of the system. The freeze-out will be delayed. Temperature and energy density decrease slower. Enhancement of entropy production will increase the production of final multiplicity since the two can be related. Since the system takes longer to cool this will lead to an enhancement in the production of thermal signals (dileptons and photons). 


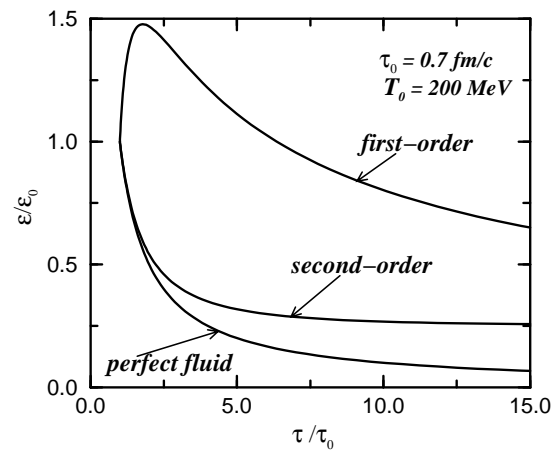

Fig. 1. The proper time evolution of energy density with initial conditions: $\tau_{0}=0.7$ $\mathrm{fm} / \mathrm{c}$ and $T_{0}=200 \mathrm{MeV}$.

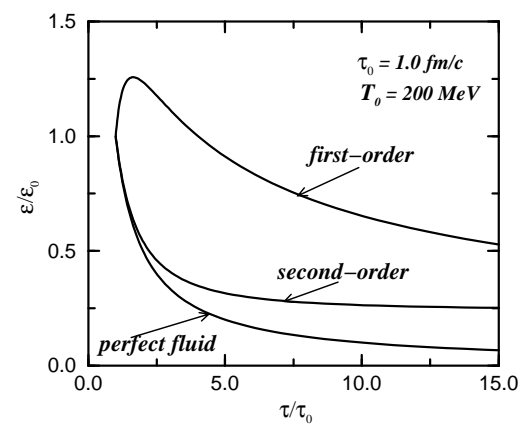

Fig. 2. The proper time evolution of energy density with initial conditions: $\tau_{0}=1.0$ $\mathrm{fm} / \mathrm{c}$ and $T_{0}=200 \mathrm{MeV}$.

\section{Conclusions}

In the early stages of collisions, non-equilibrium effects play a dominant role. Thus, nonideal fluid dynamics must be used to accurately describe the evolution of the system. Because of the undesirable features of parabolic theories, it is better to use the hyperbolic theories. Unlike in the first-order theories, where the transport equations are just the algebraic relations between the dissipative fluxes and the thermodynamic forces, in the second-order theories the transport equations describe the evolution of the dissipative fluxes from an arbitrary initial sate to a final steady-state. The presence of the relaxation terms in secondorder theories makes the structure of the resulting transport equations hyperbolic and thus have well-posed initial value problems. The first challenge faced by the second-order theories is the increase in the space of thermodynamic variables which brings new coefficients, in addition to transport coefficients, in the theory of non-ideal fluid dynamics. However, these new coefficients are determined by the equation of state. Like the primary transport coefficients which are constrained by the requirement of second law of thermodynamics, they are constrained by the requirements of hyperbolicity and hence causality. Thus, in principle, one still needs to know about the transport coefficients and the equation of state in order to solve the non-equilibrium fluid dynamics problem. The second challenge involves solving the equations numerically. Finally one would like to compare the results of non-equilibrium fluid dynamics to observables. This will require an effort to solve the full system of the resulting equations numerically.

The consequences of non-ideal fluid dynamics, both first-order (if applicable) and second-order were demonstrated here using a simplistic situation. We have seen that dissipative effects will be important in the early stages of the collision dynamics. A more careful study of the effect of the non-ideal fluid dynamics on the observables is therefore 


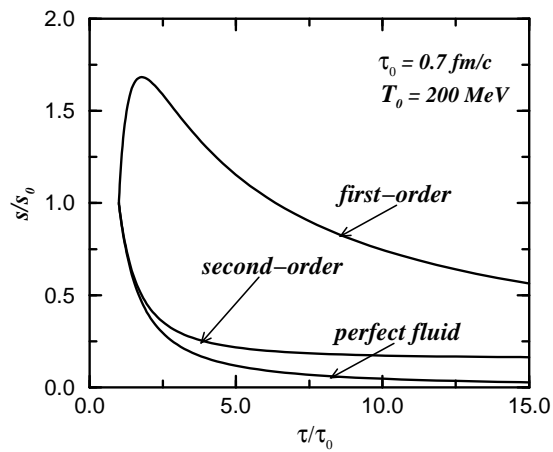

Fig. 3. The proper time evolution of entropy density with initial conditions: $\tau_{0}=$ $0.7 \mathrm{fm} / \mathrm{c}$ and $T_{0}=200 \mathrm{MeV}$.

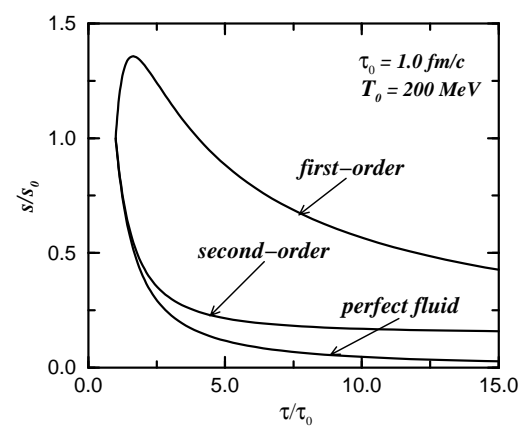

Fig. 4. The proper time evolution of entropy density with initial conditions: $\tau_{0}=$ $1.0 \mathrm{fm} / \mathrm{c}$ and $T_{0}=200 \mathrm{MeV}$.

important. Conversely, measurements of the observables related to thermodynamic quantities would allow us to determine the importance and strength of dissipative processes in heavy-ion collisions.

Here we have used only the truncated version of the transport equations and a simple equation of state. A more realistic situation will require careful analysis of both the transport coefficients and the equation of state which are employed in the full set of the equations (including the terms omitted here). It is then that one may have a better understanding of when to use either of these theories in the context of relativistic heavy ion collisions. The study of non-ideal or non-equilibrium fluid dynamics will be important for constructing hydro-molecular dynamic schemes [ 22]. A resulting hydro-molecular dynamic scheme can then be compared to the studies of multi-fluid dynamics [23].

\section{Acknowledgements}

I would like to thank the organizers of the 17th Winter Workshop on Nuclear Dynamics for inviting me to give this presentation. I would also like to thank the participants for their comments and especially for the interesting and motivating discussions with S. Gavin. I would like to thank J.I. Kapusta for reading the manuscript and valuable comments. I would also like to thank D.H. Rischke, A. Dumitru, S.A. Bass for valuable comments. This work was supported by the US Department of Energy grant DE-FG02-87ER40382.

\section{References}


1. See, for instance: Quark-Gluon Plasma, R.C. Hwa (Editor), (Advanced Series on Directions in High Energy Physics, Vol. 6, World Scientific, New Jersey, 1990).

2. H. Stöcker and W. Greiner, Phys. Rep. 137 (1986) 227; R. B. Clare and D. Strottman, Phys. Rep. 141 (1986) 177.

3. K. Kajantie, M. Kataja, L. McLerran and P.V. Russskanen, Phys. Rev. D 34 (1986) 2746; U. Katscher, D.H. Rischke, J.A. Maruhn, W. Greiner, I.N Mishustin and L.M. Satarov, Z. Phys. A346 (1993) 209.

4. J.I. Kapusta, Phys. Rev. C 24 (1981) 2545.

5. C. Eckart, Phys. Rev. 58 (1940) 919.

6. L. D. Landau and E. M. Lifshitz, Fluid Mechanics, (Addison-Wesley, Reading, Massachusetts, 1959).

7. K. Kajantie, Nucl. Phys. A418 (1984) 41c; G. Baym, Nucl. Phys. A418, (1984) 525c; A. Hosoya and K. Kajantie, Nucl. Phys. B250 (1985) 666; P. Danielewicz and M. Gyulassy, Phys. Rev. D 31 (1985) 53; L. Mornas and U. Ornik, Nucl. Phys. A587 (1995) 828; H. Kouno, Maruyama, F. Takagi and K. Saigo, Phys. Rev. D 41 (1990) 2903.

8. S. Weinberg, Gravitation and Cosmology: Principles and Applications of the General Theory of Relativity (J. Willey and Sons, New York, 1972).

9. C.W. Misner, K.S. Thorne, and J.A. Wheeler, Gravitation, (W.H. Freeman and Company, New York, 1973).

10. L.P. Csernai, Introduction to Relativistic Heavy Ion Collisions (J. Wiley an Sons, New York, 1994).

11. S.R. de Groot, H.A. van Leeuven and C.G. van Weert, Relativistic Kinetic Theory (North-Holland, Amsterdam, 1980).

12. D.H. Rischke, in Hadrons in Dense Matter and Hadrosynthesis, J. Cleymans, H.B. Geyer and F.G. Scholtz (Eds.), (Lecture Notes in Physics, Vol. 516, Springer, 1999).

13. H. Grad, Commun. Pure Appl. Math. 2 (1949) 331.

14. I. Müller, Z. Phys. 198 (1967) 329.

15. W. Israel, Ann. Phys. (N.Y.) 100 (1976) 310; J.M. Stewart, Proc. Roy. Soc. A357 (1977) 59; W. Israel and J.M. Stewart, Ann. Phys. (N.Y.) 118 (1979) 341.

16. W.A. Hiscock and L. Lindblom, Ann. Phys. (N.Y.) 151 (1983) 466; Phys. Rev. D 31 (1985) 725; ibid. 35 (1987) 3723.

17. S. Chapman and T.G. Cowling, The Mathematical Theory of Non-Uniform Gases, Third Edition (Cambridge University Press, Cambridge, 1970); W. Israel, J. math. Phys. 4 (1963) 1163; W.A. van Leeuwen and S.R. de Groot, Physics 51 (1971) 1.

18. C. Cattaneo, C.R. Acad. Sci. (Paris) 247 (1958) 431.

19. J.D. Bjorken, Phys. Rev. D 27 (1983) 140.

20. M. Prakash, M. Prakash, R. Venugopalan and G. Welke, Phys. Rep. 227 (1993) 321.

21. A. Muronga, nucl-th/0104064, submitted to Phys. Rev. Lett.

22. S.A. Bass, and A. Dumitru, Phys. Rev. C 61 (2000) 064909.

23. J. Brachmann, A. Dumitru, J.A. Maruhn, H. Stöcker, W. Greiner and D.H. Rischke, Nucl. Phys. A619 (1997) 468. 